\title{
Statistics of Turbulence Induced by Magnetic Field
}

\author{
A.A. Ferreira, M.V.A.P. Heller, M.S. Baptista, and I.L. Caldas \\ Instituto de Física, Universidade de São Paulo \\ C. P. 66318, CEP 05315-970 São Paulo, S.P., Brasil
}

Received on 26 June, 2001

\begin{abstract}
Using the TCABR tokamak facility, we analyze turbulent electrostatic fluctuations in a stationary toroidal magnetoplasma, created by radio-frequency waves and confined by two different toroidal magnetic fields. The increase of toroidal magnetic field leads to gradients in the mean plasma radial profiles and the onset of electrostatic turbulence. For the turbulent fluctuations, we show that the statistics of data collected using fixed sampling time is the same than the statistics of the time in which measurements of the data return to a specified reference interval of values. With these statistical analyses we find special invariant probability distributions, power-scaling laws for some average quantities, and long-range correlation for their oscillations. These observations suggest that turbulence has recurrent properties, as those observed in recurrent fully chaotic low-dimensional systems. Therefore, evolution of measurements of low-dimensional dynamical systems can be used to describe the recurrence observed in the tokamak edge turbulence.
\end{abstract}

\section{Introduction}

Fluctuations excited in plasma physics can lead to turbulence $[1,2]$. Experimental works carried out on a linear device showed that drift waves can destabilize the plasma and generate a turbulent spectrum [3]. Another experimental investigation showed that turbulence develops in a toroidal magnetoplasma due to drift waves desestabilization by a large magnetic confinement field [4]. On the other hand, in another experiment in a toroidal device without plasma current, anomalous tra nsport was associated to intermittent coherent structures [5].

The onset of temporal electrostatic turbulence was experimentally investigated in a stationary toroidal magnetoplasma, created by radio frequency waves [6], confined by a toroidal magnetic field. To characterize turbulence and intermittency, spectral analyses were applied to floating potential and ion saturation current fluctuations obtained with electric probes at the plasma edge. The increase of magnetic field leads to gradients in the mean pl asma radial profiles and a continuous power spectrum of higher frequency waves coupled to the driven radio frequency waves.

The understanding of chaos in deterministic systems suggests that a probabilistic description of turbulence can be applied to describe the previous results [7]. Here, we use a tool of analysis that measures the recurrence of the turbulent data, in accordance with structures varying on space-time [8]. This tool gives information about the statistics of the turbulent oscillations. Moreover, we use a recurrent measure of chaotic dynamics, the first Poincaré return time $[8,9]$ to simulate the statistical behavior of the turbulent electrostatic measurements. The applied dynamical system theory explains also the existence of scaling laws for the average values of data distributions.

\section{Experimental Data}

The experiment [6] was performed with a hydrogen magnetized circular plasma in the toroidal device of the TCABR tokamak (major radius $R_{0}=0.610 \mathrm{~m}$ and minor radius $a=0.175 \mathrm{~m}$. The stationary plasma was obtained by a $(16 \mathrm{kHz})$ radio-frequency oscillator with pulse length of $25 \mathrm{~ms}$. Hydrogen pressure $10^{-4} \mathrm{~Pa}$. Typically obtained plasma edge parameters were $T<30 \mathrm{eV}$ and $n<5 x 10^{16} \mathrm{~m}^{-3}$. In order to study the turbulence onset we applied two different toroidal magnetic fields, namely, $B_{\varphi}=0.04 \mathrm{~T}$ and $1.00 \mathrm{~T}$.

The data were collected from a multipin Langmuir probe that measures the floating potential and the ion saturation current fluctuations, mean density, electron temperature, and plasma potential. $\mathrm{T}$ he probe signals were digitally recorded at a sampling frequency of 1 $\mathrm{MHz}$. Here, we analyze the ion saturation current fluctuations, $I$, for intervals of $20 \mathrm{~ms}$ during the recorded pulses, with frequencies higher than $20 \mathrm{kHz}$.

Density and temperature for the magnetic field of $1 \mathrm{~T}$ were $n \approx(2-7) \times 10^{17} \mathrm{~m}^{-3}$ and $T_{e}=(12-30) \mathrm{eV}$. In the scrape-off-layer, the radial decay coefficients are $\lambda_{n}=-n / \nabla n \approx 2.9 \times 10^{-2} \mathrm{~m}$, for density, and $\lambda_{T_{e}}=$ $-T_{e} / \nabla T_{e} \approx 4.0 \times 10^{-2} \mathrm{~m}$ for electron temperature. 
Figs. 1(a)-(b) show samples of fluctuating ion saturation current, $I$, at $r / a=0.85$ for the two applied magnetic fields, $B_{\varphi}=1.00 \mathrm{~T}(\mathrm{a})$ and $B_{\varphi}=0.04 \mathrm{~T}(\mathrm{~b})$. The fluctuation amplitude increases with the magnetic field. Fig. 2 presents the frequency power spectra of the same fluctuations for magnetic fields of $1 \mathrm{~T}$ and $0.04 \mathrm{~T}$. This figure shows a continuous broad band of frequencies from $20 \mathrm{kHz}$ to $60 \mathrm{kHz}$ (the $16 \mathrm{kHz}$ frequency of the radio-frequency oscillator does not appear in these figures). For all frequencies the fluctuation amplitudes increase with the magnetic field.
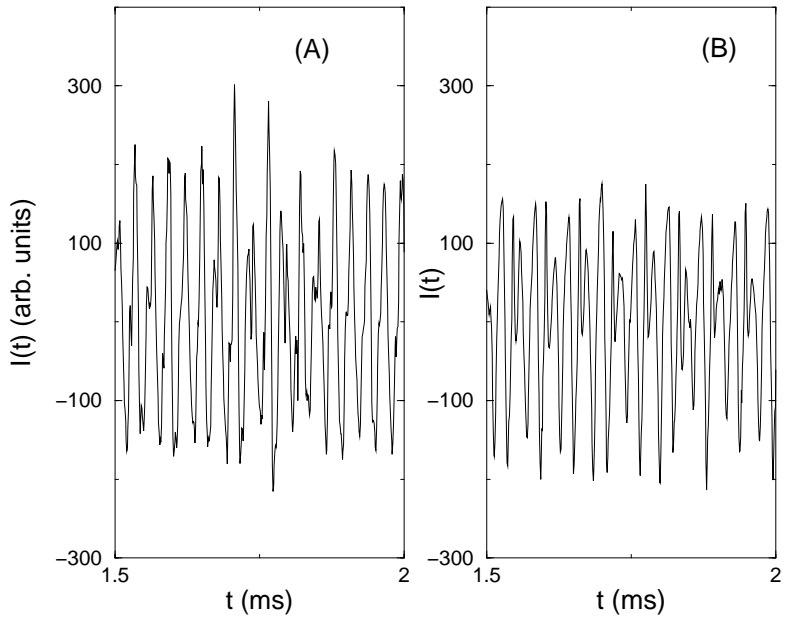

Figure 1. Samples of fluctuating ion saturation current, $I$, at $r / a=0.85$ for the two applied magnetic fields, $B_{\varphi}=1.00$ $\mathrm{T}(\mathrm{a})$ and $B_{\varphi}=0.04 \mathrm{~T}(\mathrm{~b})$

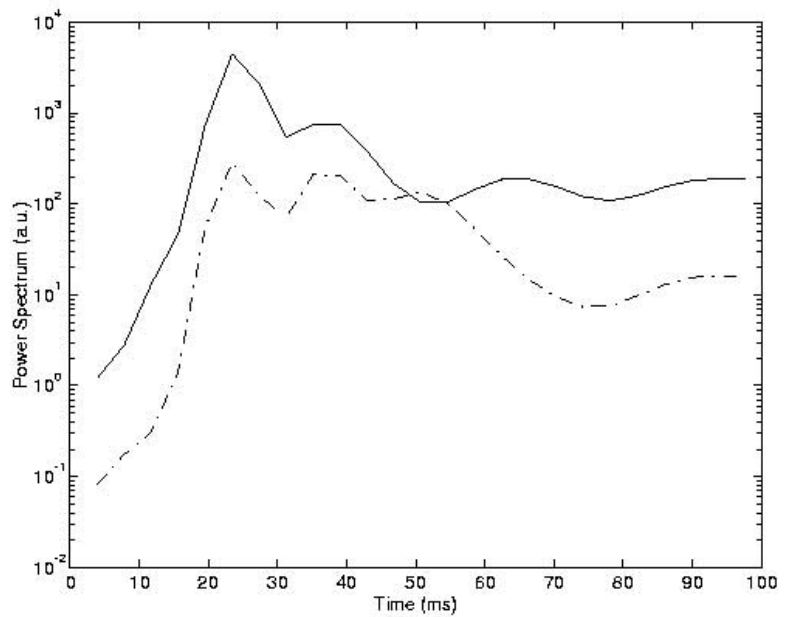

Figure 2. The frequency power spectra of the same fluctuations for magnetic fields of $1 \mathrm{~T}$ (higher amplitude power spectra) and $0.04 \mathrm{~T}$ (lower amplitude power spectra).

\section{Statistical Analysis of Turbu- lence}

We define $I_{n}$ for the value of the fluctuating ion saturation current $I(n \tau)$ at the time $t=n \tau$, where $\tau$ is the sampling rate. Fig. 3 shows the evolution of the difference

$$
R_{n}=I_{n+1}-I_{n} .
$$

These fluctuating differences are recurrent, i.e., their amplitudes eventually come back to a reference interval of values with size of $2 \delta$ at $\chi=0$. Next, in this figure, we define the returning time, $T_{n}$, as the interval of time in which $R_{n}$ repeats a value inside the chosen reference interval. The procedure to obtain these returning time is illustrated in Fig. 3, where we show a schematic representation of the $T_{n}$. The probability distribution of $T_{n}$ in normalized units $\rho\left(T_{n}\right)$, obtained for the turbulent fluctuations, can be seen in Fig. 4. For $B=1 \mathrm{~T}$ the distribution corresponds to a Poisson (Fig. 4a) [9]. However, the distribution of Fig. 4b, for $B=0.04 \mathrm{~T}$, is not a Poisson distribution.

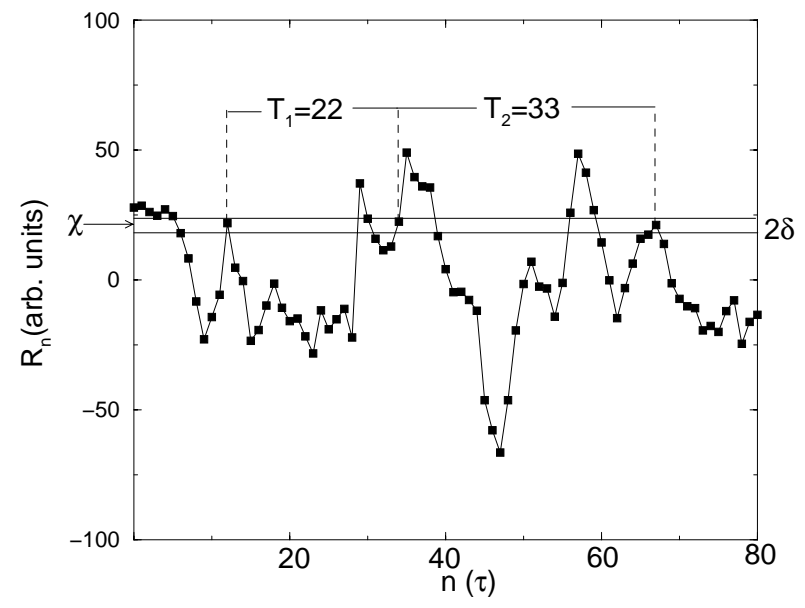

Figure 3. The difference $R_{n}$ and the returning time $T_{n}$ for the fluctuation to return to the interval of size $2 \delta$.
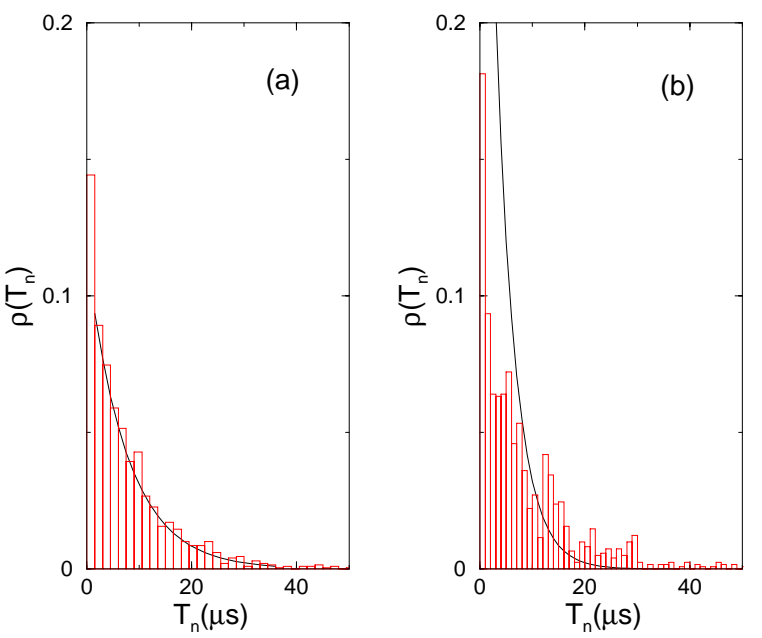

Figure 4. The probability distribution of $T_{n}$ in normalized units $\rho\left(T_{n}\right)$, obtained for the turbulent fluctuations with $1.00 \mathrm{~T}(\mathrm{a})$ and $0.04 \mathrm{~T}(\mathrm{~b})$.

The average return time $\left\langle T_{n}\right\rangle$ depends on the width $2 \delta$ of the reference interval and on the position $\chi$ 
of this interval. Figs. 5 and 6 show these dependence for the two magnetic fields. For $\chi=0$, the exponential decay of $\langle T\rangle$ with $\delta$, for small $\delta$, is almost the same for these two fields (Fig. 5). So this variation is not sensitive to the increase of turbulence. However, for the high magnetic field, $\left\langle T_{n}>\right.$ increases exponentially with $\chi$, but this variation is not exponential for the low magnetic field (Fig. 6).

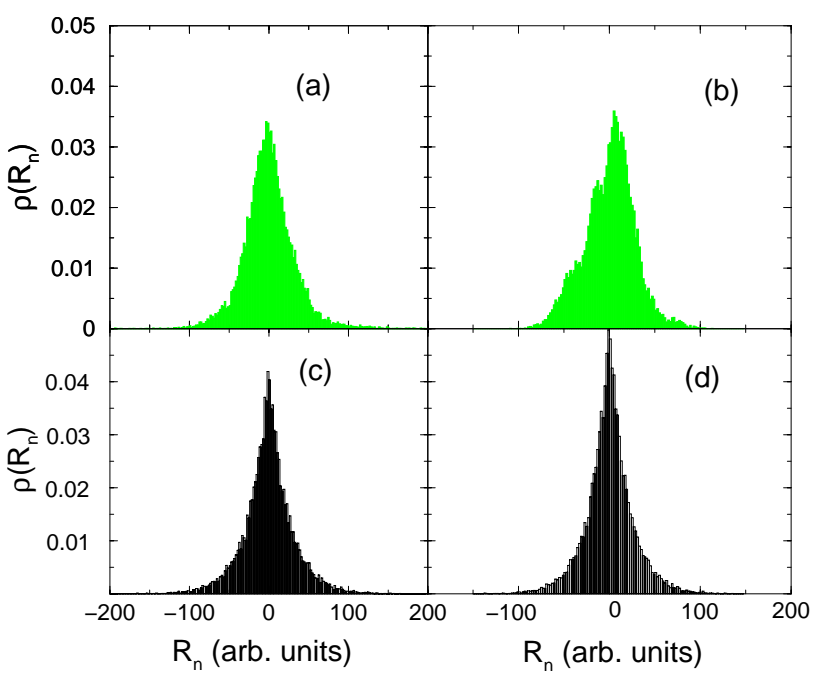

Figure 5. The exponential decay of $\langle T\rangle$ with $\delta$, for small $\delta$, with $\chi=0$.

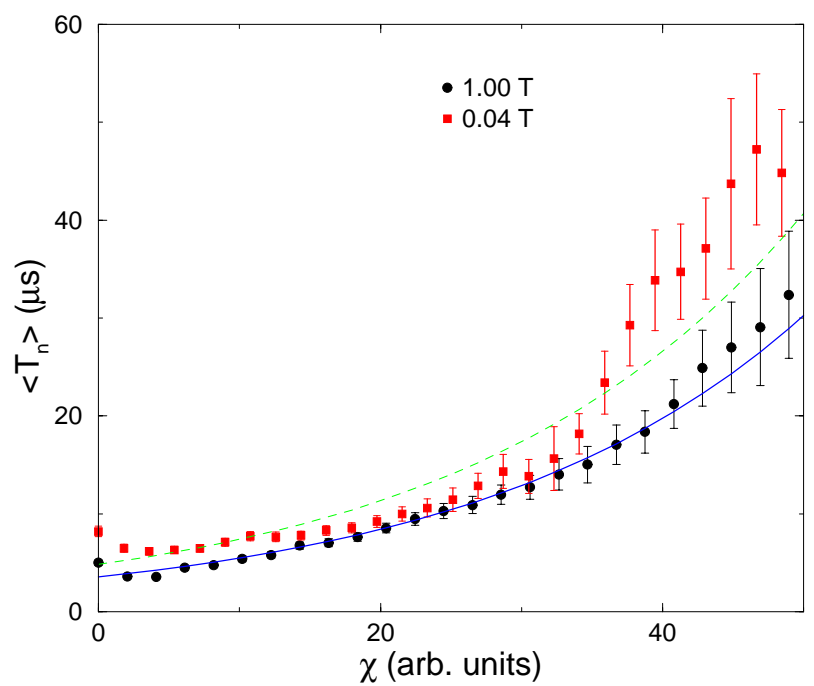

Figure 6. The exponential law of $\left\langle T_{n}\right\rangle$ in respect with $\chi$ for the $1.00 \mathrm{~T}$. For the lower field, the law between $\left\langle T_{n}\right\rangle$ and $\chi$ is not exponential.

On the other hand for the series $R_{n}$ we obtain its probability distribution $\rho\left(R_{n}\right)$ shown in Figs. $7(\mathrm{a}-\mathrm{b})$. These distributions can be represented as [9]

$$
\left.\rho\left[R_{n}(\tau)\right]=\frac{1}{2<R_{n}^{+}>} \exp ^{-\left(\left|R_{n}-<R_{n}>\right| /<R_{n}^{+}>\right.}\right),
$$

which corresponds to a sum of two Poisson distributions, where $\left\langle R_{n}>\right.$ is the average of the $R_{n}$ 's and $R_{n}^{+}$ represents $R_{n}$ bigger than $\left\langle R_{n}\right\rangle$. This Poisson-like is characterized by the average width of the distribution which is equal to $\left\langle R_{n}^{+}\right\rangle$. Figs. $7(\mathrm{c}-\mathrm{d})$ show the simulation of the distributions of Figs. 7(a-b). Next, we show how to simulate these distributions using the recurrence of a low-dimensional deterministic model.

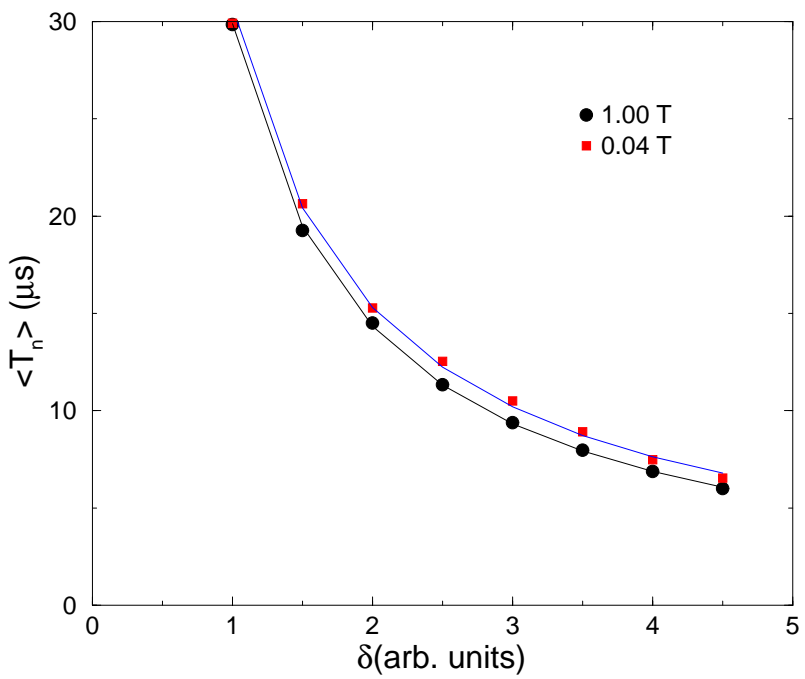

Figure 7. Probability distribution of the difference $R_{n}$ for $1.00 \mathrm{~T}(\mathrm{a})$ and $0.04 \mathrm{~T}(\mathrm{~b})$. The probability distribution of a combination of the Poincaré first return time of a chaotic trajectory $(\mathrm{c}-\mathrm{d})$.

We simulate the reported statistical behavior using a low-dimensional deterministic model, namely the logistic map $[11,12]$.

$$
x_{n+1}=b x_{n}\left(1-x_{n}\right)
$$

with the control parameter $b=4$. The recurrence is introduced through the first Poincaré return time, $P_{n}$, of the chaotic orbit, i.e., the number of map iterations required for the orbit to reach twice a specified small interval of width $2 \epsilon[10]$. Fig. 7c shows that the experimental distribution of Fig. 7a is reproduced by the distribution of values calculated for $R_{n}$ given by a linear combination of two first Poincaré return time:

$$
R_{n}=P_{n}\left(\epsilon, x_{0}\right)-P_{n}\left(\epsilon, x_{0}^{\prime}\right)
$$

noindent where $x_{0}$ and $x_{0}^{\prime}$ are two different initial conditions of the chaotic trajectory. Using the same procedure to obtain Fig. 7b we obtain Fig. 7d that does not reproduce so well the experimental distribution.

Recurrence appears in statistical analyses of fluctuation difference, $R_{n}=I_{n+1}-I_{n}$, collected using fixed sampling time, as in Figs. 7(a-b), and also in analyses obtained from the return time to a specified reference interval of $I_{n}$ (Fig. 4).

Fig. 8 shows, for both magnetic fields, the variations of the average width of the Poisson-like distribution, $\left\langle R_{n}^{+}>\right.$, obtained each $1 \mu \mathrm{s}$, along the plasma 
discharge, for previous time intervals of $250 \mu \mathrm{s}$. The width changes in time for the turbulent data of the higher magnetic field and is more stable for the lower magnetic field.

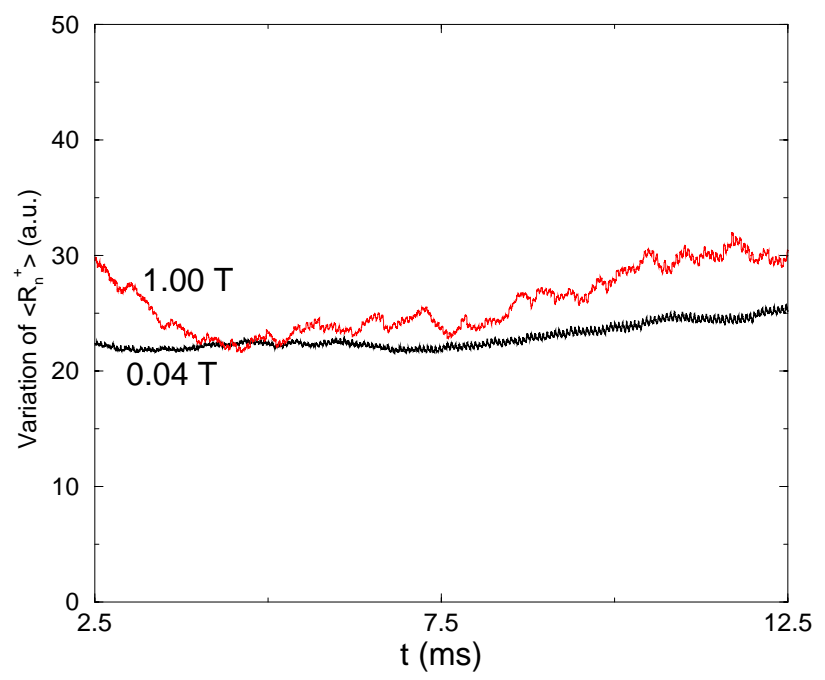

Figure 8. The variations of the average width of the Poissonlike distribution, $\left\langle R_{n}^{+}\right\rangle$, obtained each $1 \mu \mathrm{s}$, along the plasma discharge, for previous time intervals of $250 \mu \mathrm{s}$.

\section{Conclusions}

Increasing the toroidal magnetic field, we observe gradients in the plasma profiles. Spectral components, with frequencies higher than those injected in the plasma, are excited generating broader continuous frequency spectra that indicate the onset of turbulence.

We present evidences that, increasing the magnetic field, characteristics of the probability distribution of the plasma fluctuations approach those obtained by statistical analyses of the first Poincaré return time of the logistic map. Thus, two different f luctuation regimes and the transition between them are identified. In the turbulent regime, for $1 \mathrm{~T}$, the fluctuation is recurrent and its statistics is the one presented by a fully chaotic dynamic system. However, the fluctuation observed before the onset of turbulence has another kind of statistics.

\section{Acknowledges}

This work was partially supported by Brazilian governmental agencies FAPESP, CNPq, and CAPES.

\section{References}

[1] R. D. Hazeltine, J. D. Meiss, Plasma Confinement, Addison-Wesley Publishing Company, Redwood City (1992).

[2] W. Horton, Rev. Mod. Phys. 71, 735 (1999).

[3] U. Kauschke, G. Oelerich-Hill and A. Piel, Phys. Fluids B 2, 38 (1990).

[4] C. Riccardi, D. Xuantong, M. Salierno, L. Gamberale, and M. Fontanesi, Phys. Plasmas 4, 3749 (1997).

[5] F. J. Oynes, O. M. Olsen, H. L. Pécseli, A. Fredriksen, and K. Ripdal, Phys. Rev. E 57, 2242 (1998).

[6] A. A. Ferreira, M. V. A. P. Heller, I. L. Caldas, Phys. Plasmas 7, 3567 (2000).

[7] U. Frisch, Turbulence, Cambridge Press, Cambridge (1995).

[8] M. S. Baptista, I. L. Caldas, Physica A 284, 348 (2000).

[9] I. L. Caldas, M. S. Baptista, C. S. Baptista, A. A. Ferreira e M. V. A. P. Heller, Physica A 287, 91 (2000).

[10] V. Afraimovich, Chaos 7, 12 (1997).

[11] K. T. Alligood, T. Sauer, J. A. Yorke, Chaos, an Introduction to Dynamical Systems, Springer-Verlag, New York (1997).

[12] E. Ott, Chaos in Dynamical Systems, Cambridge University Press, Cambridge (1993). 